Rev. Elev. Méd. vét. Pays trop., 1977, 30 (1) : 11-18.

\title{
Contribution à l'étude de l'épidémiologie des principales trématodoses des animaux domestiques dans la région du fleuve Niger
}

\author{
par P. TAGER-KAGAN (*) \\ (avec la collaboration technique de DJIBO GARBA $\left({ }^{*}\right)$ et HAROUNA ALMOUMOUNE $\left({ }^{*}\right)$ )
}

\begin{abstract}
RÉSUMÉ
Des enquêtes malacologiques ont permis de mettre en évidence, le long du fleuve Niger et dans des mares de sa valtée, des gastéropodes appartenant aux genres Lymnaea, Biomphalaria et Bulinus ; l'évolution des populations de mollusques au cours de l'année a été étudiée.

L'élevage de mollusques au Laboratoire a permis de préciser le mode et la durée des cycles parasitaires de Fasciola gigantica, de Paramphistomum microbothrium, de Gastrodiscus aegyptiacus.

Une étude de la réinfestation parasitaire après traitement a été faite sur 3 troupeaux pâturant le long du fleuve.
\end{abstract}

\section{INTRODUCTION}

A la suite d'enquêtes effectuées dans la région du fleuve Niger (12) chez divers ruminants et équidés, il est apparu que les affections parasitaires provoquées par les trématodes tenaient une place importante dans la pathologie parasitaire des animaux domestiques.

Ces helminthes ont un cycle biologique complexe qui nécessite à un certain moment le passage obligatoire par un mollusque pulmoné d'eau douce basommatophore; aussi, dans un programme de lutte contre ces trématodoses, il s'avérait nécessaire d'en étudier l'épidémiologie. Dans ce but, des recherches au niveau de l'hôte intermédiaire, du parasite et de l'hôte définitif ont été entreprises.

(*) Laboratoire de l'Elevage, B. P. 485, Niamey, Rép. du Niger.

\section{PRINCIPAUX TREMATODES RENCONTRÉS}

SUR LES ANIMAUX DOMESTIQUES AU NIGER (11)

\section{A. Bovins}

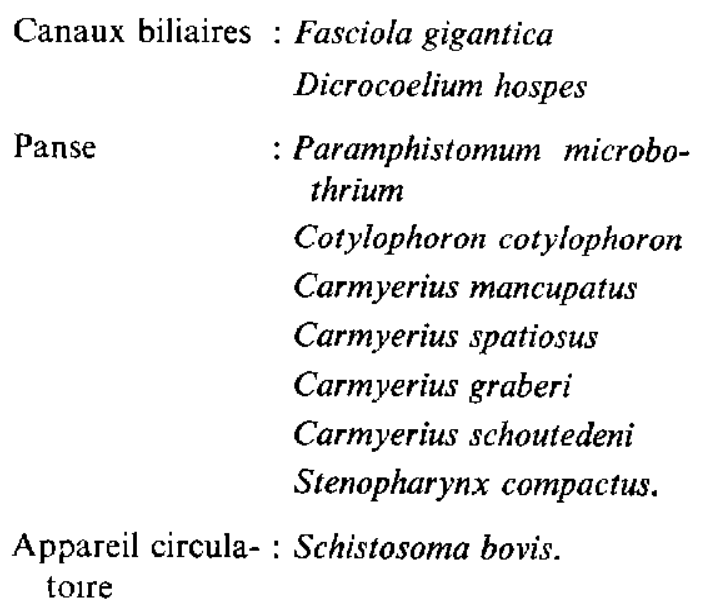




\section{B. Ovins - Caprins}

Canaux biliaires : Fasciola gigantica

Panse

: Paramphistomum microbothrium.

\section{Equins}

Gros intestin : Gastrodiscus aegyptiacus.

\section{ENQUÊTES MALACOLOGIQUES}

1) Liste et lieux de récolte des mollusques

La plupart des enquêtes malacologiques ont été effectuées sur les rives du fleuve Niger et dans les mares permanentes et semi-permanentes de sa vallée ; à l'encontre des travaux de S. GRETILLAT (7) qui n'a pas mis en évidence dans le fleuve Niger de faune malacologique vectrice de trématodoses, et qui de ce fait nie l'intervention du fleuve dans leur épidémiologie, nous avons trouvé les mollusques suivants :

\section{A. Gasteropodes pulmonés basommatophores}

- Famille des Lymnaeidae

Genre Lymnaea (Lamarck, 1799)

Limnae natalensis (Krauss, 1848)

$$
1-2 \text { - } 3-4-5-6 \text {. }
$$

- Famille des Planorbidae

Genre Biomphalaria (Preston, 1910)

Biomphalaria pfeifferi (Krauss, 1848) 7 - 5 - 4 .

Genre Segmentorbis (Mandahl-Barth, 1954)

Segmentorbis kanisaensis (Preston)

9.

Genre Afrogyrus (Blainville, 1826)

Afrogyrus coretus

9.

\section{- Famille des Bulidae}

Genre Bulinus (Muller, 1781)

Bulinus jousseaumi (Dautznberg, 1890) $8-3-1$.

Bulinus truncatus rohlfsi (Clessin, 1886) 18 - 1 - 19 - 20-2 - 3 - 11 - 12 - 10 - 21 - 13 14.

Bulinus forskalii (Ehrenberg, 1831) $6-9-1-10-4-5-7-11-12$ - $13-14-15$ $16-17-18-20$.

Bulinus umbilicatus (Mandalh-Barth) 2 - 9.

\section{B. Gasteropodes prosobranches}

Genre Bellamaya (Olivier)

Bellamaya unicolor

$9-7$.

Genre Cleopatra (Morelet)

Cleopatra bulimoides senegalensis $1-2$ - 3.

Genre Laniste (Peters)

Laniste ovum

$$
7 \text { - } 4 \text {. }
$$

2) Variation de la population de mollusques dans l'année

\section{- Limnaea natalensis}

Sur les mares permanentes et semi permanentes, les mollusques commencent à réapparaître en octobre ; leur population est importante de janvier à avril avec un maximum en févriermars.

A partir de la mi-avril, les populations diminuent sensiblement.

Sur le fleuve Niger, dans les criques où existent une végétation de Nymphea, de Sphenoclea, des limnées apparaissent fin janvier, début février. Les populations restent peu importantes; elles atteignent leur maximum en mars-avril, puis elles décroissent rapidement selon le moment où le retrait des eaux intervient sur la végétation.

\section{- Biomphalaria pfeifferi}

Sur les mares permanentes ou semi permanentes, ces mollusques commencent à se développer vers la fin août ; l'accroissement des populations est maximal entre novembre et février.

Sur le fleuve Niger, il n'a jamais été trouvé de Biomphalaria; paradoxalement, les élevages au Laboratoire de Biomphalaria pfeifferi dans des cuvettes remplies d'eau du fleuve se sont révélés florissants.

\section{- Bulinus forskalii}

Dans les mares, les bulins font leur apparition fin août, début septembre, leur développement est maximal durant les mois d'octobre à décembre. A partir de janvier, les populations décroissent rapidement.

Sur le fleuve, les mollusques se développent plus tardivement ; leur population, peu importante en décembre, commence à s'accroître en 


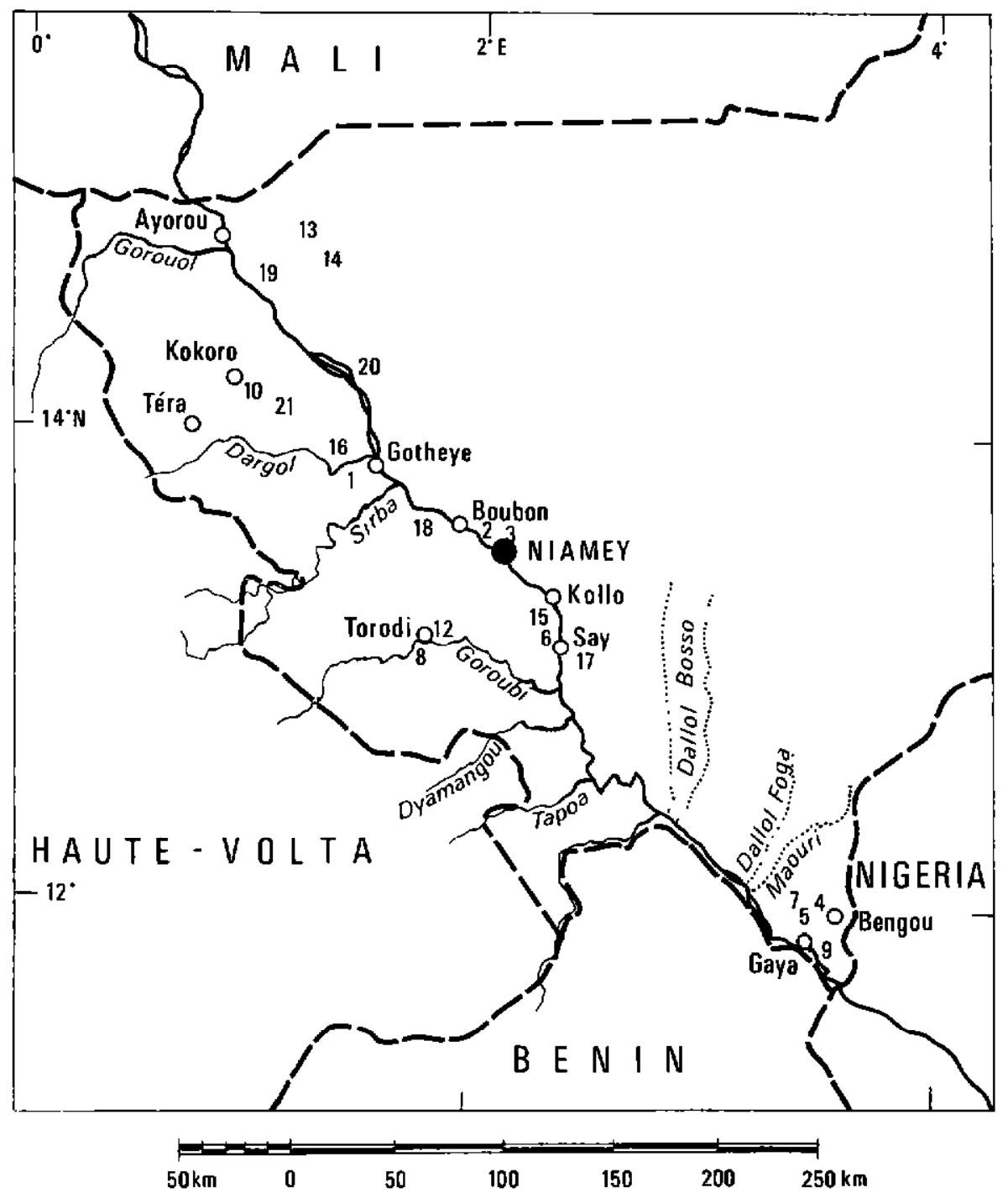

Carte de la région du fleuve Niger et liste des lieux de récolte des mollusques.

\begin{tabular}{|c|c|c|c|c|c|c|c|c|c|c|}
\hline Gotheye & (Fleuve) & 7 & Sabon Bırnı & (mare) & 12 & Kobadié & (mare) & 17 & Seberı & (mare) \\
\hline Boubon & (Fleuve) & 8 & Torodi & (mare) & 13 & Tem & (mare) & 18 & Namaro & (Fleuve) \\
\hline Rio bravo & & 9 & Gaya & (Fleuve) & 14 & Gabou & (mare) & 19 & Ayorou & (Fleuve) \\
\hline Kawara & (mare) & 10 & Kocoro & (mare) & 15 & Kollo & (mare) & 20 & Sekoira & Fleuve) \\
\hline Say & (Fleuve) & 11 & Sagagorou & (mare) & 16 & Dargol & (mare) & 21 & Namga & (mare). \\
\hline
\end{tabular}

janvier pour attendre un maximum en févriermars.

Dans les mares résiduelles de la vallée du fleuve se formant au moment du retrait des eaux, se développent des petites colonies de bulins qui dureront jusqu'à l'assèchement des collections d'eau.

\section{- Bulinus truncatus rohlfsi}

Dans les mares, les mollusques commencent à se multiplier fin oct obre-début novemtre Un accroissement massif des populations est observé en janvier-février. A partir de mai, le nombre de bulins baisse considérablement.

Sur les rives du fleuve, les populations de bulins se développent vers le mois de décembre ; elles atteignent leur maximum d'accroissement entre les mois de février et avril. 


\section{3) Dissection des mollusques récoltés}

a) Gastéropodes pulmonés d'eau douce, vecteurs de maladie parasitaire (1).

\begin{tabular}{ll}
\hline \multicolumn{1}{c}{ Mollusques } & \multicolumn{1}{c}{ Parasites transmis } \\
\hline Limnaea natalensis & Fasciola gigantica \\
\hline Biomphalaria pfeifferi & $\begin{array}{l}\text { Schistosoma mansoni } \\
\text { Paramphistomum sukari }\end{array}$ \\
\hline Bulinus jousseaumi & Schistosoma curassoni \\
\hline Bulinus truncutus rohlfsi & $\begin{array}{l}\text { Schistosoma haematobium } \\
\text { Schistosoma curassoni } \\
\text { Schistosoma bovis } \\
\text { Paramphistomum microboth- } \\
\text { rium }\end{array}$ \\
\hline Bulinus forskalii & $\begin{array}{l}\text { Schistosoma hematobium } \\
\text { Paramphistomum phillerouxi } \\
\text { Stephanopharyn compactus } \\
\text { Gastrodiscus aegyptiacus }\end{array}$ \\
\hline
\end{tabular}

b) Dissection des mollusques (6)

\section{Limnaea natalensis.}

Les limnées renfermaient :

- des cercaires de Fasciola gigantica (récoltées en $1-2-3-4-18$ ).

Le maximum d'infestation des mollusques par les cercaires se situe durant les mois de mars et d'avril ;

- des Xiphidiocercaires (récoltées à 6-5); ce sont des cercaires de distome à queue élancée dont l'extrémité antérieure est armée d'un stylet. Ces cercaires sont les formes larvaires de digénes appartenant aux familles des Dicrocoelidés, Plagiorchidés et Haplometridés ;

- des Furcocercaires (récoltées à 4).

\section{Biomphalaria pfeifferi}

A la dissection des mollusques on a trouvé :

- des Furcocercaires (récoltés à 7 - 4).

$\mathrm{Ce}$ sont les formes larvaires de Trematodes appartenant aux Schistosomidés et aux Strigeïdés ;

- des Xiphidiocercaires (récoltés à 5-7); 7).

\section{Bulinus truncatus rohlfsi}

Chez ces bulins ont été reconnus :

- des furcocercaires (récoltés à 12 - 13 - 14 $21-1-2-3)$;

- des cercaires de type echinostome (région antérieure pourvue d'un collier épineux) (récoltés à $12-13)$;

— des Xiphidiocercaires (récoltés à 11 - 13 10) ;

- des cercaires de type amphistome (récoltés à $13-21-1-2-3-18-9-20$ ).

\section{Bulinus forskalii}

On a trouvé dans ces mollusques :

- des furcocercaires (récoltés à 7-11-4 $6-12)$;

- des Xiphidiocercaires (récoltés à 7 - 11 4) ;

- des cercaires de type amphistome (14 - 4 $7-1-2-3-18-20$ ).

\section{III. ÉleVAGe De MOllusques. ÉTUDE EXPÉRIMENTALE DES CYCLES PARASITAIRES}

\section{1) Elevage de mollusques $(1,12)$}

\section{- Méthode générale}

Des cuvettes en plastique de $30 \mathrm{~cm} \times 10 \mathrm{~cm}$, d'une capacité d'environ 41 sont remplies d'eau provenant du fleuve Niger; ces cuvettes sont laissées dans le Laboratoire de Parasitologie sur les paillasses ou sur le sol. Elles reçoivent plusieurs mollusques de la même espèce recueillis au cours des prospections malacologiques.

La nourriture est à base de feuilles de salade ; l'eau des cuvettes est changée 2 fois par semaine.

Les mollusques nouveau-nés sont retirés de la cuvette des parents pour être mis dans une cuvette " nursery"

Ce sont ces mollusques qui servent lors des expériences sur le cycle parasitaire des différents Trématodes.

\section{- Résultats}

Limnées.

Des limnées récoltées en octobre, après une dizaine de jours d'acclimatement aux nouvelles conditions de vie, commencent à pondre. 
Les pontes se succéderont jusqu'en marsavril.

Un pourcentage assez élevé d'œufs ne se développe pas; nous pensons que le $\mathrm{pH}$ du fleuve $(5$ à 5,5$)$ est un obstacle à l'éclosion des œufs, ce qui semblerait expliquer la densité assez faible des limnées dans les différents gîtes découverts le long du fleuve.

A partir de mai, on observe des mortalités importantes dans les cuvettes (température de l'eau atteignant $35^{\circ}$ ).

\section{Biomphalaria pfeifferi}

Des Biomphalaria mis dans des cuvettes fin août commencent à pondre vers la mi-septembre.

Les pontes et développement des œufs sont importants jusqu'en février-début mars, puis les pontes diminuent. A partir de mai, l'élevage périclite.

\section{Bulinus forskalii}

Ce sont les mollusques qui s'accommodent le plus difficilement aux conditions de vie imposées au laboratoire; un grand nombre de bulins meurent lors de la phase d'acclimatation. Cependant, on observe des pontes à partir de septembre; de jeunes mollusques apparaissent encore fin avril alors que dans la nature les populations décroissent à partir de janvier.

\section{Bulinus truncatus rohlfsi}

C'est l'espèce qui s'accommode le mieux aux conditions d'élevage. Les bulins récoltés en octobre commencent à pondre peu de temps après. Les pontes se succèdent jusqu'en janvierfévrier.

A partir de mai, les conditions délevage (température de l'eau trop importante) font péricliter les populations.

\section{ETUDE DU CYCLE DES PRINCIPAUX TRÉMATODES}

\section{1) Méthode générale}

a) Récolte des œufs et mise en incubation.

Des parasites adultes sont récoltés vivants chez l'hôte définitif; ils sont laissés 24 h dans une boîte de pétri remplie de sérum physiolo- gique, au bout de ce temps les parasites sont retirés, les cufs pondus sont lavés à l'eau, puis remis dans une boîte de pétri avec de l'eau du robinet.

Ces boîtes sont laissées à la température ambiante jusqu'à l'éclosion des miracidia.

b) Infestation des hôtes intermédiaires.

Des jeunes mollusques, nés au Laboratoire, donc indemnes de toute infestation, sont mis au contact de miracidia.

Au bout d'une dizaine de jours, puis tous les 4 jours, on dissèque un mollusque pour observer l'évolution de l'infestation (Sporocyste, redies, cercaire).

c) Récolte des métacercaires.

L'élevage des mollusques se faisant dans des cuvettes, on applique à l'intérieur de celles-ci, sur le pourtour, un morceau de plastique sur lequel les cercaires après leur sortie des mollusques viennent s'enkyster.

Lorsqu'on estime le nombre de métacercaires enkystés suffisant, on retire le plastique que l'on garde pendant quelques jours dans un récipient d'eau afin de laisser «mûrir » les métacercaires.

Après avoir déterminé le nombre, on les décolle du plastique avec un pinceau et on les fait absorber à l'hôte définitif pour clore le cycle.

\section{2) Résultats}

\section{A) Fasciola gigantica $(3,2)$}

a) Développement de l'œuf

Les cufs mettent entre 15 et 25 jours pour donner des miracidia.

b) Chez l'hôte intermédiaire (Limnaea natalensis)

Après infestation par le miracidium, on trouve des rédies au bout de 12 à 20 jours dans la Limnée.

Des cercaires libres dans le mollusque apparaissent au bout de 36-50 jours.

Des cercaires sont émises par le mollusque au bout de 40-57 jours.

De l'œuf au stade métacercaire, il s'écoule de 55 à 80 jours. 


\section{c) Chez l'hôte définitif}

Un bovin infesté par 2000 métacercaires laisse apparaître dans ses fèces des cufs de Fasciola gigantica au bout de 95 jours. (L'autopsie du bovin sacrifié révéla un foie truffé de jeunes Fasciola venant juste d'atteindre leur maturité sexuelle.)

Il faut donc environ 6 mois pour qu'un ouf de Fasciola gigantica donne chez un bovin une Fasciola adulte.

B) Paramphistomum microbothrium $(4,5,7,8)$

a) Développement de l'œuf

Les cufs mettent de 13 à 19 jours pour donner des miracidies.

b) Infestation de l'hồte intermédiaire

\section{1) Bulinus rohlfsi}

Après infestation par le miracidium, on trouve des rédies au bout de 19 à 20 jours.

Des rédies contenant des cercaires apparaissent vers le $33^{\mathrm{e}}$ jour.

Des cercaires sont émises vers le $41^{\mathrm{e}}$ jour.

De l'œuf au stade métacercaire, il s'écoule 60 jours environ.

\section{2) Bulinus forskalii}

Après infestation par le miracidium, on trouve des rédies au bout de 22 à 23 jours.

Des cercaires sont émises au bout de 35 à 36 jours.

De l'œuf au stade métacercaire, il s'écoule de 48 à 52 jours.

\section{c) Chez l'hôte définitif}

Un jeune bovin, indemne en paramphistome, a reçu per os environ 1000 métacercaires; des examens coproscopiques périodiques ont laissé apparaître des œufs de Paramphistome au bout de 73 jours. Il faut donc environ 130 jours pour que le cycle de Paramphistomum microbothrium soit accompli.

\section{C) Gastrodiscus aegyptiacus (9)}

a) Développement de l'æuf

Les œufs mettent de 6 à 9 jours pour donner des miracidia.

\section{b) Hôte intermédiaire (Bulinus forskalii)}

Après l'infestation par le miracidium, des rédies apparaissent entre le $10^{\mathrm{e}}$ et le $20^{\mathrm{e}}$ jour.

On trouve des cercaires libres dans le mollusque vers le $30^{\circ}$ jour.

De l'œuf au stade métacercaire, il s'écoule de 37 à 40 jours.

c) Chez l'hôte définitif

Un âne infesté par des métacercaires a rejeté dans ses fèces des œufs de Gastrodiscus aegyptiacus au bout de 143 jours.

Il faut donc environ 6 mois pour qu'un œuf de Gastrodiscus donne chez l'hôte défnitif un parasite adulte.

\section{ETUDE \\ DE LA RËINFESTATION PARASITAIRE APRẼS UN TRAITEMENT}

\section{1) Troupeau laitier de Say}

Ce troupeau a été traité le 28 février 1975 avec du Wormyl 10 (Association de Disto 5 et de Thiabendazole).

\section{a) Fasciola gigantica}

L'apparition d'cufs de Fasciola gigantica dans les fèces des bovins a été constatée début juillet; comme il faut en moyenne 95 jours pour qu'un métacercaire donne une Fasciola adulte, on peut estimer que l'infestation des animaux a eu lieu début avril 1975.

A partir de janvier 1975 (Loukia, mares laissées par la crue du fleuve) les limnées se sont multipliées; les bovins pâturant en janvier le long du fleuve et porteurs de Fasciola ont évacué dans leurs fèces des æufs de douve.

Ces œufs, après transformation en miracidia, ont infesté les populations de limnées existant à cette époque. Ces limnées ont donné des cercaires 55 jours à 80 jours après leur infestation par le miracidium, c'est-à-dire en mars-avril ; c'est à cette époque que les bovins du troupeau laitier de Say ont dû s'infester.

\section{b) Paramphistomes}

L'apparition d'œufs de Paramphistome dans les fèces des bovins a été constatée fin juindébut juillet ( 120 jours après le déparasitage). 
Comme il faut en moyenne 75 jours pour qu'un métacercaire donne un Paramphistome adulte, on peut estimer que l'infestation des animaux a eu lieu fin avril-début mai 1975.

Des bovins porteurs de Paramphistomes sont venus pâturer, début février le long du fleuve et des mares (laissées par le fleuve au moment de sa décrue); les œufs de Paramphistomes évacués dans les fèces de ces animaux, après transformation en miracidium ont infesté les Bulinus truncatus rohlfsi et Bulinus forskalii se multipliant à cette époque. Comme il faut de 50 à 60 jours pour le développement des formes infestantes de Paramphistome chez ces bulins, les mollusques ont libéré des cercaires fin marsdébut avril, ce qui correspond à l'infestation observée sur le troupeau de Say.

Le même processus d'infestation en Fasciola gigantica et en Paramphistomes a été constaté sur-un troupeau de la région d'Ayorou. Les animaux traités au Wormyl le 20/02/75 ont rejeté des œufs de Paramphistome et de Fasciola au cours du mois de juillet.

\section{2) Troupeau de Gaya}

Ce troupeau a été traité le 5 février 1975 avec du Wormyl 10.

L'apparition d'œufs de Paramphistomidae dans les fèces a été constatée fin mai, avec un taux plus important vers juillet-août.

Des infestations expérimentales ont montré qu'il fallait de 70 à 80 jours pour obtenir un helminthe mature à partir d'une métacercaire. Les bovins se sont donc infestés à la mi-février avec un pic d'infestation en métacercaires en avril-mai.

Cinquante à 60 jours s'écoulant du stade œuf à celui de métacercaire, l'infestation par les bovins à la mi-février correspondrait à une émission de cercaires par des mollusques infestés par des miracidia vers la mi-décembre, ce que corrobore la constatation de Bulinus forskalii contenant des formes larvaires de Paramphistomidae dans les mares aux alentours de Gaya à la mi-décembre.

Les infestations des bovins en métacercaires en avril-mai correspondraient à des émissions de cercaires par des mollusques infestés en févriermars, ce qui concorde avec les populations importantes de Bulinus rohlfsi trouvées à cette époque dans les mares et de Bulinus forskalii sur les rives du fleuve.

\section{CONCLUSION}

Dans le but d'une prophylaxie des trématodoses atteignant les animaux domestiques, des enquêtes épidémiologiques ont été menées qui ont permis de connaître la répartition et la variation de population des hôtes intermédiaires au cours de l'année dans la région du fleuve Niger. Des élevages de mollusques au Laboratoire nous ont permis de préciser le mode et la durée de quelques cycles parasitaires.

Les processus épidémiologiques sont conditionnés par la coexistence de 3 éléments qui sont :

- les animaux (réceptifs et source de parasites) ;

- les conditions de survie des formes exogènes des parasites;

- les facteurs assurant l'infestation des animaux.

Aussi pour établir un calendrier de traitement de ces trématodoses, il nous faut connaître le moment et les lieux où ces 3 éléments sont réunis ; dans ce but, une étude des mouvements de troupeaux au cours de l'année doit être faite.

Nous tenons à remercier le Professeur MANDALH-BARTH (Charlottenlund Danemark) qui a bien voulu déterminer les mollusques récoltés au cours de nos enquêtes.

\section{SUMMARY}

Contribution to the epidemiological study of the principal domestic animal trematodosis in the Niger river area

Some investigations about freshwater snails along the Niger river and the pools of the valley have allowed to show off snails belonging to the genus Lymnaea, Biomphalaria and Bulinus; development of snails population has been studied during one year. 
Breeding of snails in Laboratory has allowed us to specify the parasitic cycle of Fasciola gigantica, Paramphistomum microbothrium and Gastrodiscus aegyptiacus.

Studies on the parasitic infestation after treatment have been carried out on three droves feeding along the Niger river.

\section{RESUMEN}

\section{Contribución al estudio de la epidemiologia de las principales trematodosis} de los animales domesticos en la region del rio Niger

Encuestas malacologicas permitieron evidenciar a lo largo del rio Niger y en charcas de su cuenca gasterópodos perteneciendo a los generos Lymnaea, Biomphalaria y Bulinus; Se estudia la evolución de las poblaciones de moluscos durante el año.

La cria de moluscos en el Laboratorio permitió determinar el modo y la duración de los ciclos parasitarios de Fasciola gigantica, de Paramphistomum microbothrium, de Gastrodiscus aegyptiacus.

Se ha estudiado la reinfestación parasitaria después de tratamiento en 3 rebaños pastoreando a to largo del rio.

\section{BIBLIOGRAPHIE}

1. BIRGI (E.), GRABER (M.). Mollusques pulmonés d'eau douce basommatophores, vecteurs au Tchad d'affections parasitaires du bétail ; leur élevage au laboratoire. Rev. Elev. Méd. vét. Pays trop., 1969, 22. (3) : 393-408.

2. BITAKARAMIRE (P. K.). Lymnaea natalensis laboratory culture and production of Fasciola gigantica metacercariae. Parasitology, 1968, 58 (3) : 653656.

3. DAYNES (P.). La distomatose à Madagascar. Cycle de Fasciola gigantica. Rev. Elev. Méd. vét. Pays trop., $1967,20(4): 557-562$.

4. DINNIK (J. A.). The snails hosts of certain Paramphistomidae and Gastrothylacidae (Trematoda) discovered by the late Dr. P. L. Le Roux in Africa. $J$. Helminth., 1965, $39(2-3): 141-150$.

5. DINNIK (J. A.), DINNIK (N. N.). The life cycle of Paramphistomum microbothrium. Fischoeder 1901 (Trematoda, Paramphistomidae). Parasitology, 1954, 44 (3-4) : 285-299.

6. Fain (A.). Contribution à l'étude des formes larvaires des Trematodes au Congo Belge. Mémoires, Inst. r. Colon. Belge - Sect. Sci. Nat. Med. coll. in $8^{\circ}$, Tome 22, fasc. 5 .
7. GRETILLAT (S.). Communication au $3^{e}$ Congrès international de Parasitologie (München, août 1974) parue dans les P-V de ce Congrès ref., 21-B 21.

8. LENGY (J.). Study on Paramphistomum microbothrium (Fischoeder 1901), a rumen parasite of cattle in Israël. Bull. Res. Counc. Israël, 1960, 9B : 71-130.

9. LE ROUX (P. L.). Life cycle of Gastrodiscus aegyptiacus (Cobbold, 1876). Trans. r. Soc. trop. Med. Hyg., 1958, 52 (1) : 14-15.

10. MANDALH-BARTH (G.). Key to identification of east and central african freshwater snails of medical and veterinary importance. Bull. Wld Hlth. Org., 1962, 27 (1) : 135-150.

11. TAGER-KAGAN (P.). Helminthes et helminthiases des animaux domestiques au Niger. Rapport. Niamey, Laboratoire de l'Élevage, 1974.

12. TIBAYRENC (R.). Enquête helminthologique sur le fleuve Niger. Rapport Niamey, Laboratoire de l'Elevage, 1971 .

13. WARDS (P. A.), TRAVIS (D.), RUE (R. E.). Methods of establishing and maintening snails in the laboratory. Nat. Inst. Hith. Bull., 1947 (189) : 170. 